UDC 519.63

Вестник СПбГУ. Прикладная математика. Информатика... 2020. Т. 16. Вып. 2

MSC 35F10, 65D30, 70F05

\title{
Estimates for Taylor series method to linear total systems of PDEs
}

\author{
L. K. Babadzanjanz, I. Yu. Pototskaya, Yu. Yu. Pupysheva
}

St. Petersburg State University, 7-9, Universitetskaya nab., St. Petersburg, 199034, Russian Federation

For citation: Babadzanjanz L. K., Pototskaya I. Yu., Pupysheva Yu. Yu. Estimates for Taylor series method to linear total systems of PDEs. Vestnik of Saint Petersburg University. Applied Mathematics. Computer Science. Control Processes, 2020, vol. 16, iss. 2, pp. 112-120.

https://doi.org/10.21638/11701/spbu10.2020.203

A large number of differential equations can be reduced to polynomial form. As was shown in a number of works by various authors, one of the best methods for the numerical solution of the initial value problem for such ODE systems is the method of Taylor series. In this article we consider the Cauchy problem for the total linear PDE system, and then - a theorem about the accuracy of its solutions by this method is formulated and proved. In the final part of the article, four examples of total systems of partial differential equations to the well-known two-body problem are proposed: two of them are related to the Kepler equation, one to the motion of a point in the orbit plane, and the last to the motion of the orbit plane.

Keywords: Taylor series method, total linear PDE system, polynomial system, numerical PDE system integration.

Introduction. Issues considering in this article are: the formulation of the Cauchy problem for total systems of partial differential equations including polynomial and linear; the Taylor series method; local error estimation for linear total Cauchy problem. As examples, we consider four total polynomial systems to the elliptical two body problem.

Initial value problem (IVP or Cauchy problem for polynomial and linear total systems). Consider the total system of partial differential equations [1] with the initial conditions

$$
\frac{\partial x_{j}}{\partial t_{\nu}}=f_{\nu, j}\left(x_{1}, \ldots, x_{n}, t_{1}, \ldots, t_{s}\right), \quad x_{j}\left(t_{0}\right)=x_{0, j}, \quad j=1, \ldots, n, \quad \nu=1, \ldots, s .
$$

Numerical methods for solving this problem are oriented to the general case when the right-hand sides $f_{\nu, j}$ belong to the class of smooth or piecewise smooth functions. At the same time, in many applied problems, for which numerical methods are developed, it is quite possible to reduce the problem (1) to the case when the functions $f_{\nu, j}$ are algebraic polynomials in $x_{1}, \ldots, x_{n}$ (by introducing the special additional variables [2, 3]). In these cases, the obtained Cauchy problem is called polynomial, and it can be written as

$$
\begin{gathered}
\frac{\partial x_{j}}{\partial t_{\nu}}=\sum_{m \in[1: L+1]} \sum_{i \in I(m)} a_{\nu, j, m}[i] x^{i}, \quad x_{j}\left(t_{0}\right)=x_{0, j}, \quad j=1, \ldots, n, \quad \nu=1, \ldots, s, \quad(2) \\
x=\left(x_{1}, \ldots, x_{n}\right) \in C^{n}, \quad i=\left(i_{1}, \ldots, i_{n}\right), x^{i}=x_{1}^{i_{1}} \ldots . x_{n}^{i_{n}}, x_{j}, x_{0, j}, t_{\nu}, t_{0, \nu}, a_{\nu, j, m} \in C, \\
|i|=i_{1}+\ldots+i_{n}, \quad I(m)=\left\{i \in Z^{n}\left|i_{1}, \ldots, i_{n} \geqslant 0,\right| i \mid=m\right\}, \quad L \in[0:+\infty) .
\end{gathered}
$$

This is IVP to total system of polynomial PDEs (or the total polynomial Cauchy problem). For small $x_{1}, \ldots, x_{n}$ the equations (1) one often linearizes and utilizes as first approximations. In what follows we will write down the linear problem as

(C) Санкт-Петербургский государственный университет, 2020 


$$
\begin{gathered}
\frac{\partial x}{\partial t_{\nu}}=a_{\nu}+A_{\nu} x, \quad x\left(t_{0}\right)=x_{0}, \quad \nu=1, \ldots, s, \\
x=\left(x_{1}, \ldots, x_{n}\right), \quad x_{0}=\left(x_{0,1}, \ldots, x_{0, n}\right) \in C^{n}, \\
a_{\nu}=\left(a_{\nu, 1}, \ldots, a_{\nu, n}\right) \in C^{n}, \quad\left|a_{\nu}\right|=\max _{i \in[1: n]}\left|a_{i}\right|, \\
t=\left(t_{1}, \ldots, t_{s}\right), \quad t_{0}=\left(t_{0,1}, \ldots, t_{0, s}\right) \in C^{s}, \quad A_{\nu}=\left(a_{\nu, i, j}\right), \quad a_{\nu, i, j} \in C,
\end{gathered}
$$

denote its solution by $x\left(t, t_{0}, x_{0}\right)$ or $x(t)$. In addition, we will utilize the designations

$$
\begin{gathered}
x^{(k)}=\frac{\partial^{|k|} x}{\partial t^{k}}, \quad|k|=k_{1}+\ldots+k_{s}, \quad x_{0}^{(k)}=x^{(k)}\left(t_{0}\right), \\
x^{(0)}=x, \quad x_{0}^{(0)}=x_{0}, \quad|x|=\max _{i \in[1: n]}\left|x_{i}\right|, \quad O_{\rho}\left(t_{0}\right)=O_{\rho_{1}}\left(t_{0}\right) \times \ldots \times O_{\rho_{s}}\left(t_{0}\right), \\
O_{\rho_{\nu}}\left(t_{0}\right)=\left\{t \in C^{s}\left|(\forall j \in[1: s], j \neq \nu)\left(t_{j}=t_{j, 0}\right),\right| t_{\nu}-t_{0, \nu} \mid<\rho_{\nu}\right\}, \\
T_{M} x\left(t, t_{0}, x_{0}\right)=\sum_{m=0}^{M} x_{0}^{(m)} \frac{\left(t-t_{0}\right)^{m}}{m !}, \quad \delta T_{M} x\left(t, t_{0}, x_{0}\right)=x\left(t, t_{0}, x_{0}\right)-T_{M} x\left(t, t_{0}, x_{0}\right), \\
m !=\prod_{\mu=1}^{s} m_{\mu} !, \quad 0 !=1, \quad k=\left(k_{1}, \ldots, k_{s}\right), \\
M=\left(M_{1}, \ldots, M_{s}\right) \in[0:+\infty)^{s}, \quad \rho=\left(\rho_{1}, \ldots, \rho_{s}\right) \in(0,+\infty)^{s},
\end{gathered}
$$

where $T_{M}$ and $\delta T_{M}$ are the operators that put in correspondence the Taylor polynomial $T_{M} x\left(t, t_{0}, x_{0}\right)$ and the remainder $\delta T_{M} x\left(t, t_{0}, x_{0}\right)$ to the solution of the problem (3). We denote as $R\left(t_{0}, x_{0}\right)=\left(R_{1}\left(t_{0}, x_{0}\right), \ldots, R_{s}\left(t_{0}, x_{0}\right)\right), R_{\nu}\left(t_{0}, x_{0}\right)$, the vector radius of convergence of the Taylor series and, instead, later in this paper as a domain where Taylor series converge we will utilize $O_{\rho}\left(t_{0}\right)=O_{\rho_{1}}\left(t_{0}\right) \times \ldots \times O_{\rho_{s}}\left(t_{0}\right)$, see above in $(4)$ and below in Proposition).

On the Taylor series method. The Taylor series method [4-8] for solving the Cauchy problem (3) consists in constructing a table of approximate values $x_{t_{w}}=x\left(t_{w}\right)$ using the formula

$$
x_{\tau_{w}}=T_{N_{w}} x\left(\tau_{w}, \tau_{w-1}, x_{\tau_{w-1}}\right), \quad w=1,2, \ldots,
$$

here

$$
\begin{gathered}
N_{w}=\left(N_{w, 1}, \ldots, N_{w, s}\right) \in(0: \infty)^{s}, \quad \tau_{0}=t_{0}, \tau_{w}=\tau_{w-1}+h_{w}, \\
\tau_{w}=\left(\tau_{w, 1}, \ldots, \tau_{w, s}\right), \quad h_{w}=\left(h_{w, 1}, \ldots, h_{w, s}\right) \in C^{s}
\end{gathered}
$$

and $h_{w}$ has to satisfy the inequalities

$$
\left|h_{w, \nu}\right|<R_{\nu}\left(\tau_{w-1}, x_{\tau_{w-1}}\right), \quad \nu=1, \ldots, s .
$$

The calculation of each value of $\tilde{x}_{\tau_{w}}$ is called the step of the method, and $h_{w}$ is called the size of this step (or, briefly, the step). In the general case of integration along a curve in $C^{s}$ all $h_{w, \nu}$ are complex numbers, and points $\tau_{w}$ lie on this curve. To calculate $\tilde{x}_{\tau_{w}}$ for some given $\tau_{w}$ with high accuracy by formula (5), even for $\tau_{w}$ from its domain of convergence (see (5)), the number of steps may turn out to be large, which can cause a fast accumulation of rounding errors and an increased processor time. That is why it is advisable to use the steps as large as possible (in actual fact, one has to find all $\rho_{\nu}$ as large as possible see (6) and Proposition). 


\section{Local error estimation for linear total Cauchy problem.}

Estimates. Now we turn to problem (3). In addition to (4), we will use also the notation

$$
\begin{gathered}
\left(A_{\nu}^{k_{\nu}} x\right)_{i}=\sum_{j=1}^{n} a_{\nu, i, j} x_{j}, \quad \rho_{\nu}=1 / s_{\nu}, \quad s_{\nu}=\left\|A_{\nu}\right\|_{\infty}=\max _{i \in[1: n]} s_{\nu, i}, \quad s_{\nu, i}=\sum_{j=1}^{n}\left|a_{\nu, i, j}\right|, \\
T_{\mu} e^{\tau}=\sum_{m=0}^{\mu} \frac{\tau^{m}}{m !}, \quad \delta T_{\mu} e^{\tau}=e^{\tau}-T_{\mu} e^{\tau}, \quad \mu=1,2, \ldots
\end{gathered}
$$

Proposition. The solution $x\left(t, t_{0}, x_{0}\right)$ of the problem $(3)$ is holomorphic on $O_{\rho_{\nu}}\left(t_{0}\right)$ separately in $t_{\nu}$ and satisfies there the inequality

$$
\left|\delta T_{M} x\left(t, t_{0}, x_{0}\right)\right| \leqslant\left(\left|x_{0}\right|+\left|a_{\nu}\right| \rho_{\nu}\right) \delta T_{M_{\nu}} e^{\left|t_{\nu}-t_{0, \nu}\right| / \rho_{\nu}} .
$$

Proof. Because of

$$
k=\left(k_{1}, \ldots, k_{s}\right), \quad \frac{\partial^{|k|} x}{\partial t^{k}}=\frac{\partial^{k_{\nu}} x}{\partial t^{k_{\nu}}} \Rightarrow x^{k_{\nu}}=A_{\nu}^{k_{\nu}} x+A_{\nu}^{k_{\nu}-1} a_{\nu}, \quad\left|\left(A_{\nu}^{k_{\nu}} x\right)_{i}\right| \leqslant|x| \rho_{\nu}^{k_{\nu}},
$$

then

$$
\begin{gathered}
\left|\delta T_{M} x\left(t, t_{0}, x_{0}\right)\right|=\left|\sum_{l=M_{\nu}}^{+\infty}\left(A_{\nu}^{l} x+A_{\nu}^{l-1} a_{\nu}\right)\left(t_{\nu}-t_{0, \nu}\right)^{l} / l !\right| \leqslant \\
\leqslant\left(\left|x_{0}\right|+\left|a_{\nu}\right| \rho_{\nu}\right) \sum_{l=M_{\nu}+1}^{+\infty}\left|\left(t_{\nu}-t_{0, \nu}\right) / \rho_{\nu}\right|^{l} / l !=\left(\left|x_{0}\right|+\left|a_{\nu}\right| \rho_{\nu}\right) \delta T_{M_{\nu}} e^{\left|t_{\nu}-t_{0, \nu}\right| / \rho_{\nu}},
\end{gathered}
$$

which is the required result.

Improving estimates: scaling transformations and choice of scaling factors. The smaller $s_{\nu}=\rho_{\nu}^{-1}$, the better the estimates (8). In order to be able to improve these estimates, it is natural to introduce a scaling transformation in (3):

$$
x_{j}=\alpha_{j} y_{j}, \quad \alpha_{j}>0, \quad j \in[1: n] .
$$

In connection with (9), we write down the Cauchy problem

$$
\begin{gathered}
\frac{\partial y}{\partial t_{\nu}}=b_{\nu}+B_{\nu} y, \quad y\left(t_{0}\right)=y_{0}, \quad \nu=1, \ldots, s, \\
y=\left(y_{1}, \ldots, y_{n}\right), \quad y_{0}=\left(y_{0,1}, \ldots, y_{0, n}\right), \quad b_{\nu}=\left(b_{\nu, 1}, \ldots, b_{\nu, n}\right), \quad B_{\nu}=\left(b_{\nu, i, j}\right), \\
y_{i}=\alpha_{i}^{-1} x_{i}, \quad b_{\nu, i}=\alpha_{i}^{-1} a_{\nu, i}, \quad b_{\nu, i, j}=\alpha_{i}^{-1} \alpha_{j} a_{\nu, i, j},
\end{gathered}
$$

and will use the designations (see $(7))$ :

$$
\rho_{\nu}(\alpha)=\frac{1}{s_{\nu}(\alpha)}, \quad s_{\nu}(\alpha)=\max _{i \in[1: n]} s_{\nu, i}(\alpha), \quad s_{\nu, i}(\alpha)=\alpha_{i}^{-1} \sum_{j=1}^{n} \alpha_{j}\left|a_{\nu, i, j}\right|, \quad \alpha=\left(\alpha_{1}, \ldots, \alpha_{n}\right) .
$$

Using (8), one can easily prove that Proposition implies.

Corollary. The solution $x\left(t, t_{0}, x_{0}\right)$ of the problem (3) is holomorphic on $O_{\rho_{\nu}(\alpha)}\left(t_{0}\right)$ (see (4)) separately in $t_{\nu}$ and satisfies there the inequality

$$
\left|\delta T_{M} x_{i}\left(t, t_{0}, x_{0}\right)\right| \leqslant \alpha_{i}\left(\left|y_{0}\right|+\left|b_{\nu}\right| \rho_{\nu}(\alpha)\right) \delta T_{M_{\nu}} e^{\left|t_{\nu}-t_{0, \nu}\right| / \rho_{\nu}(\alpha)} .
$$


Ability to select scaling factors $\alpha_{1}, \ldots, \alpha_{n}$ to reduce the value $s_{\nu}(\alpha)$ makes Corollary a real tool of automatically assigning a step size of integration with a priori guaranteed local error estimation. The use of this corollary leads to the minimax problem [6, 9-14]. For linear ODEs, we previously used the Perron's theorem $[6,9,10]$. We use it here too.

Theorem (Perron). Let the matrix $P=\left(p_{i, j}\right)$ be positive, $i$. e. $p_{i, j}>0$ for all $i, j \in[1: n]$. Then the following statements are true [12]:

a) there is a single eigenvalue $\lambda(P)$ of this matrix with the largest absolute value;

b) this eigenvalue is positive and simple, and the corresponding eigenvector can be chosen positive;

c) the following equality holds:

$$
\lambda(P)=\min _{x_{1}, \ldots, x_{n}>0} \max _{i \in[1: n]}\left(\sum_{j=1}^{n} p_{i, j} x_{j} / x_{i}\right) .
$$

$\mathrm{R}$ e $\mathrm{m}$ a $\mathrm{r} \mathrm{k}$ 1. More general Frobenius theorem and other results about eigenvalues and eigen-vectors of non-negative matrices can be found in [13].

$\mathrm{R}$ e $\mathrm{m}$ a $\mathrm{r} \mathrm{k}$ 2. With any approach to choosing scaling factors (see (9)), it is worth considering that in practical calculations it is enough to use their rough approximations with a relative error about 10 percents, since Corollary remains true for any positive scale factors, on the one hand, and, on the other hand, their small change (e. g. in the second valid digit) will not lead to a noticeable deterioration in the estimate (12). In [6, 10], devoted to the method of Taylor series for polynomial ODE systems (that is, for systems (2) with $s=1$ ), it was noted that in applications it is possible to restrict ourselves with the Perron's theorem by replacing the matrix $A$ with a close matrix $A^{+}$with positive elements. Since when $s \neq 1$ the matrix $A_{\nu}$ is not square, this idea should be slightly refined: one can supplement the matrix $A_{\nu}$ with a square nonzero matrix $A_{\nu}^{\sim}=\left(\tilde{a}_{\nu, i, j}\right)$ by small modulo elements and then, instead $A_{\nu}$, use $A_{\nu}^{+}=\left(\left|\tilde{a}_{\nu, i, j}\right|\right)$. It is important here to recall that the eigenvalues of a matrix depend continuously on its elements $[14,15]$.

Thus, in applications we can assume that $\left|a_{\nu, i, j}\right|>0$ for all $i, j \in[1: n]$, and then as scaling factors $\alpha_{1}, \ldots, \alpha_{n}$ in Corollary it is natural to use the components of a positive eigenvector $\alpha^{*}=\left(\alpha_{1}^{*}, \ldots, \alpha_{n}^{*}\right)$ of the matrices $A_{\nu}^{+}=\left(\left|\tilde{a}_{\nu, i, j}\right|\right)$ corresponding to its eigenvalue $\lambda\left(A_{\nu}^{+}\right)$, maximum in absolute value.

Examples. Here we consider four total polynomial systems to the elliptic two-body problem (from the Bregman thesis [16] and paper [17]), and then Corollary can be applied to their linearized versions. First, we will consider the equations of the two-body problem and their solution in relative Cartesian coordinates centered on the point mass $m^{0}$. Next, before considering the above four total systems, we give in the form of a table all the functions and arguments used in them.

The considered examples may be of real interest to specialists in the field of mechanics, astronomy, celestial mechanics, astrometry, and astrodynamics.

The equations of the two-body problem and their solution to the elliptic case. Consider the equations of motion of a point mass $m$ in a central Newtonian field of mass $m^{0}$, using relative Cartesian coordinates centered on the point mass $m^{0}$ :

$$
\ddot{\xi}_{i}=-\mu \xi_{i} r^{-3} \quad\left(\text { or } \dot{\xi}_{i}=\eta_{i} \quad \dot{\eta}_{i}=-\mu \xi_{i} r^{-3}\right), \quad i \in[1: 3],
$$

and the general solution of these equations for the elliptic case:

$$
\xi_{i} / a=A_{i} \sqrt{1-e^{2}} \sin E+B_{i}(\cos E-e), \quad i \in[1: 3], \quad r / a=(1-e \cos E),
$$




$$
\begin{gathered}
A_{1}=-\sin \omega \cos \Omega-\cos \omega \sin \Omega \cos i, \quad B_{1}=\cos \omega \cos \Omega-\sin \omega \sin \Omega \cos i, \\
A_{2}=-\sin \omega \sin \Omega+\cos \omega \cos \Omega \cos i, \quad B_{2}=\cos \omega \sin \Omega+\sin \omega \cos \Omega \cos i,
\end{gathered}
$$

$$
A_{3}=\cos \omega \sin i, \quad B_{3}=\sin \omega \sin i
$$

$$
E-e \sin E=M, \quad M=M_{0}+n\left(t-t_{0}\right), \quad n=\sqrt{\mu / a^{3}}, \quad \mu=\gamma\left(m^{0}+m\right),
$$

where $a$ (semi-major axis), $e$ (eccentricity), $M_{0}$ (mean anomaly of the epoch $\left.t_{0}\right), \Omega$ (longitude of the ascending node), $i$ (inclination), $\omega$ (pericenter argument) are Kepler's elements (arbitrary constants), and $E$ (eccentric anomaly), $M$ (mean anomaly) are functions of time; $\gamma$ is Newtonian universal constant of gravitation.

Functions and arguments used. Next, we are going to write out four total polynomial systems: two for solving Kepler's equation and two for the coordinates and velocities of the two-body problem. For the reader's convenience, we give Table of the main functions (placed there into rect) and arguments. The four total systems mentioned above are numbered (see the column $\mathrm{N}$ in the Table) in an understandable way.

Table. Main functions and arguments

\begin{tabular}{|c|c|c|}
\hline $\mathrm{N}$ & Functions & Arguments \\
\hline 1 & $\varphi_{1}=E, \varphi_{2}=\sin E, \varphi_{3}=\cos E, \varphi_{4}=(1-e \cos E)^{-1}$ & $\tau_{1}=e, \tau_{2}=M$ \\
\hline 2 & $\varphi_{1}=E, \varphi_{2}=\sin E, \varphi_{3}=\cos E, \varphi_{4}=(1-e \cos E)^{-1}$, & $t_{1}=t, t_{2}=a$, \\
& $\varphi_{5}=a^{-1 / 2}$ & $t_{3}=e, t_{4}=M_{0}$ \\
\hline \multirow{2}{*}{3} & $\varphi_{1}=E, \varphi_{2}=\sin E, \varphi_{3}=\cos E, \varphi_{4}=(1-e \cos E)^{-1}$, & $t_{1}=t, t_{2}=a$, \\
& $\varphi_{5}=a^{-1 / 2}, \varphi_{6}=\left(1-e^{2}\right)^{1 / 2}, \varphi_{7}=\left(1-e^{2}\right)^{-1 / 2}$, & $t_{3}=e, t_{4}=M_{0}$ \\
& $\varphi_{8}=\xi_{1}, \varphi_{9}=\xi_{2}, \varphi_{10}=\xi_{3}$, & \\
& $\varphi_{11}=\eta_{1}, \varphi_{12}=\eta_{2}, \varphi_{13}=\eta_{3}$ \\
\hline & $\varphi_{14}=A_{1}, \varphi_{15}=A_{2}, \varphi_{16}=A_{3}, \varphi_{17}=B_{1}, \varphi_{18}=B_{2}$, & $t_{5}=i, t_{6}=\Omega$, \\
& $\varphi_{19}=B_{3}, \varphi_{20}=A_{4}=\sin \omega \cos i, \varphi_{21}=B_{4}=\cos \omega \cos i$, & $t_{7}=\omega$ \\
\hline \multicolumn{2}{|c|}{$\varphi_{22}=A_{5}=\sin \Omega, \varphi_{23}=B_{5}=\cos \Omega$} & \\
\hline
\end{tabular}

To apply Corollary, it remains for the user to linearize the equations in the vicinity of the initial data, then write out the matrix $A_{\nu}^{+}-\lambda I$ and, finally, find the maximum eigenvalue in absolute value and the corresponding positive eigenvector.

The first total polynomial system for Kepler's equation. Here, Kepler's equation (15) is used in order to write out a total polynomial system that is satisfied by an eccentric anomaly, considered as a function of eccentricity and mean anomaly. Assuming (see Table) $\varphi_{1}=E, \varphi_{2}=\sin E, \varphi_{3}=\cos E, \varphi_{4}=(1-e \cos E)^{-1}, \tau_{1}=e, \tau_{2}=M$ and using the equality $\varphi_{1}-\tau_{1} \sin \varphi_{1}=\tau_{2}$ as an implicit representation of $\varphi_{1}\left(\tau_{1}, \tau_{2}\right)$, we get the equations

$$
\begin{gathered}
\frac{\partial \varphi_{1}}{\partial \tau_{1}}=\varphi_{2} \varphi_{4}, \quad \frac{\partial \varphi_{1}}{\partial \tau_{2}}=\varphi_{4}, \quad \frac{\partial \varphi_{2}}{\partial \tau_{1}}=\varphi_{2} \varphi_{3} \varphi_{4}, \quad \frac{\partial \varphi_{2}}{\partial \tau_{2}}=\varphi_{3} \varphi_{4}, \quad \frac{\partial \varphi_{3}}{\partial \tau_{1}}=-\varphi_{2}^{2} \varphi_{4}, \\
\frac{\partial \varphi_{3}}{\partial \tau_{2}}=-\varphi_{2} \varphi_{4}, \quad \frac{\partial \varphi_{4}}{\partial \tau_{1}}=\varphi_{3} \varphi_{4}^{2}-\tau_{1} \varphi_{2}^{2} \varphi_{4}^{3}, \quad \frac{\partial \varphi_{4}}{\partial \tau_{2}}=-\tau_{1} \varphi_{2} \varphi_{4}^{3} .
\end{gathered}
$$

The second total polynomial system for Kepler's equation. Now we write out a total system that satisfies the eccentric anomaly $E$, considered as a function of time $t$ and three Keplerian elements $a, e, M_{0}$. Assuming 


$$
\begin{gathered}
\varphi_{1}=E, \varphi_{2}=\sin E, \varphi_{3}=\cos E, \varphi_{4}=(1-e \cos E)^{-1}, \varphi_{5}=a^{-1 / 2} \\
t_{1}=t, t_{2}=a, t_{3}=e, t_{4}=M_{0}
\end{gathered}
$$

and using equality $\varphi_{1}-t_{3} \sin \varphi_{1}=t_{4}+\sqrt{\mu} t_{2}^{-3 / 2}\left(t_{1}-t_{0}\right)$ as an implicit function $\varphi_{1}\left(t_{1}, t_{2}, t_{3}, t_{4}\right)$ representation, we get the equations

$$
\begin{gathered}
\frac{\partial \varphi_{1}}{\partial t_{1}}=\sqrt{\mu} \varphi_{4} \varphi_{5}^{3}, \quad \frac{\partial \varphi_{1}}{\partial t_{2}}=-\frac{3 \sqrt{\mu}\left(t_{1}-t_{0}\right)}{2} \varphi_{4} \varphi_{5}^{5}, \quad \frac{\partial \varphi_{1}}{\partial t_{3}}=\varphi_{2} \varphi_{4}, \quad \frac{\partial \varphi_{1}}{\partial t_{4}}=\varphi_{4}, \\
\frac{\partial \varphi_{2}}{\partial t_{1}}=\sqrt{\mu} \varphi_{3} \varphi_{4} \varphi_{5}^{3}, \quad \frac{\partial \varphi_{2}}{\partial t_{2}}=-\frac{3 \sqrt{\mu}\left(t_{1}-t_{0}\right)}{2} \varphi_{3} \varphi_{4} \varphi_{5}^{5}, \quad \frac{\partial \varphi_{2}}{\partial t_{3}}=\varphi_{2} \varphi_{3} \varphi_{4}, \quad \frac{\partial \varphi_{2}}{\partial t_{4}}=\varphi_{3} \varphi_{4}, \\
\frac{\partial \varphi_{3}}{\partial t_{1}}=-\sqrt{\mu} \varphi_{2} \varphi_{4} \varphi_{5}^{3}, \quad \frac{\partial \varphi_{3}}{\partial t_{2}}=\frac{3 \sqrt{\mu}\left(t_{1}-t_{0}\right)}{2} \varphi_{2} \varphi_{4} \varphi_{5}^{5}, \quad \frac{\partial \varphi_{3}}{\partial t_{3}}=-\varphi_{2}^{2} \varphi_{4}, \quad \frac{\partial \varphi_{3}}{\partial t_{4}}=-\varphi_{2} \varphi_{4}, \\
\frac{\partial \varphi_{4}}{\partial t_{1}}=-\sqrt{\mu} t_{3} \varphi_{2} \varphi_{4}^{3} \varphi_{5}^{3}, \quad \frac{\partial \varphi_{4}}{\partial t_{2}}=\frac{3 \sqrt{\mu}\left(t_{1}-t_{0}\right)}{2} t_{3} \varphi_{2} \varphi_{4}^{3} \varphi_{5}^{5}, \quad \frac{\partial \varphi_{4}}{\partial t_{3}}=\varphi_{3} \varphi_{4}^{2}-t_{3} \varphi_{2}^{2} \varphi_{4}^{3}, \quad(17) \\
\frac{\partial \varphi_{4}}{\partial t_{4}}=-t_{3} \varphi_{2} \varphi_{4}^{3}, \quad \frac{\partial \varphi_{5}}{\partial t_{j}}=0, \quad j=1,3,4, \quad \frac{\partial \varphi_{5}}{\partial t_{2}}=\frac{1}{2} \varphi_{5}^{3} .
\end{gathered}
$$

The first total polynomial system for the two body equations. The quantities

$$
\begin{gathered}
\varphi_{1}=E, \quad \varphi_{2}=\sin E, \quad \varphi_{3}=\cos E, \quad \varphi_{4}=(1-e \cos E)^{-1}, \\
\varphi_{5}=a^{-1 / 2}, \quad \varphi_{6}=\left(1-e^{2}\right)^{1 / 2}, \quad \varphi_{7}=\left(1-e^{2}\right)^{-1 / 2}, \\
\varphi_{7+i}=\xi_{i}, \quad \varphi_{10+i}=\eta_{i}, \quad i=1,2,3,
\end{gathered}
$$

we consider as functions of time $t_{1}=t$ and elements $t_{2}=a, t_{3}=e, t_{4}=M_{0}$ and we assume elements $\Omega, i, \omega$ as parameters. Using formulas (13)-(16) we obtain that these functions satisfy the total system of partial differential equations (the equations for $\varphi_{1}, \ldots, \varphi_{5}$ and (17) are the same):

$$
\begin{gathered}
\frac{\partial \varphi_{1}}{\partial t_{1}}=\sqrt{\mu} \varphi_{4} \varphi_{5}^{3}, \quad \frac{\partial \varphi_{1}}{\partial t_{2}}=-\frac{3 \sqrt{\mu}\left(t_{1}-t_{0}\right)}{2} \varphi_{4} \varphi_{5}^{5}, \quad \frac{\partial \varphi_{1}}{\partial t_{3}}=\varphi_{2} \varphi_{4}, \quad \frac{\partial \varphi_{1}}{\partial t_{4}}=\varphi_{4}, \\
\frac{\partial \varphi_{2}}{\partial t_{1}}=\sqrt{\mu} \varphi_{3} \varphi_{4} \varphi_{5}^{3}, \quad \frac{\partial \varphi_{2}}{\partial t_{2}}=-\frac{3 \sqrt{\mu}\left(t_{1}-t_{0}\right)}{2} \varphi_{3} \varphi_{4} \varphi_{5}^{5}, \quad \frac{\partial \varphi_{2}}{\partial t_{3}}=\varphi_{2} \varphi_{3} \varphi_{4}, \quad \frac{\partial \varphi_{2}}{\partial t_{4}}=\varphi_{3} \varphi_{4}, \\
\frac{\partial \varphi_{3}}{\partial t_{1}}=-\sqrt{\mu} \varphi_{2} \varphi_{4} \varphi_{5}^{3}, \quad \frac{\partial \varphi_{3}}{\partial t_{2}}=\frac{3 \sqrt{\mu}\left(t_{1}-t_{0}\right)}{2} \varphi_{2} \varphi_{4} \varphi_{5}^{5}, \quad \frac{\partial \varphi_{3}}{\partial t_{3}}=-\varphi_{2}^{2} \varphi_{4}, \quad \frac{\partial \varphi_{3}}{\partial t_{4}}=-\varphi_{2} \varphi_{4}, \\
\frac{\partial \varphi_{4}}{\partial t_{1}}=-\sqrt{\mu} t_{3} \varphi_{2} \varphi_{4}^{3} \varphi_{5}^{3}, \quad \frac{\partial \varphi_{4}}{\partial t_{2}}=\frac{3 \sqrt{\mu}\left(t_{1}-t_{0}\right)}{2} t_{3} \varphi_{2} \varphi_{4}^{3} \varphi_{5}^{5}, \quad \frac{\partial \varphi_{4}}{\partial t_{3}}=\varphi_{3} \varphi_{4}^{2}-t_{3} \varphi_{2}^{2} \varphi_{4}^{3}, \\
\frac{\partial \varphi_{4}}{\partial t_{4}}=-t_{3} \varphi_{2} \varphi_{4}^{3}, \quad \frac{\partial \varphi_{5}}{\partial t_{j}}=0, \quad j=1,3,4, \quad \frac{\partial \varphi_{5}}{\partial t_{2}}=\frac{1}{2} \varphi_{5}^{3}, \\
\frac{\partial \varphi_{6}}{\partial t_{j}}=\frac{\partial \varphi_{7}}{\partial t_{j}}=0, \quad j=1,2,4, \quad \frac{\partial \varphi_{6}}{\partial t_{3}}=-t_{3} \varphi_{7}, \quad \frac{\partial \varphi_{7}}{\partial t_{3}}=t_{3} \varphi_{7}^{3},
\end{gathered}
$$

Вестник СПбГУ. Прикладная математика. Информатика... 2020. Т. 16. Вып. 2 


$$
\begin{gathered}
\frac{\partial \varphi_{7+i}}{\partial t_{1}}=t_{2} \varphi_{4} \varphi_{5}^{3} \sqrt{\mu}\left(A_{i} \varphi_{6} \varphi_{3}-B_{i} \varphi_{2}\right) \\
\frac{\partial \varphi_{7+i}}{\partial t_{2}}=\left(\varphi_{3}-t_{3}\right) B_{i}+\varphi_{3} \varphi_{6} A_{i}+\frac{3}{2} \sqrt{\mu}\left(t_{1}-t_{0}\right) t_{2} \varphi_{4} \varphi_{5}^{5}\left(B_{i} \varphi_{2}-A_{i} \varphi_{3} \varphi_{6}\right) \\
\frac{\partial \varphi_{7+i}}{\partial t_{3}}=t_{2}\left(A_{i} \varphi_{3} \varphi_{2} \varphi_{4} \varphi_{6}-A_{i} t_{3} \varphi_{2} \varphi_{7}-B_{i}\left(1+\varphi_{2}^{2} \varphi_{4}\right)\right) \\
\frac{\partial \varphi_{7+i}}{\partial t_{4}}=t_{2} \varphi_{4}\left(A_{i} \varphi_{6} \varphi_{3}-B_{i} \varphi_{2}\right) \\
\frac{\partial \varphi_{10+i}}{\partial t_{1}}=-\left(\mu t_{3} \varphi_{2} \varphi_{4}^{3} \varphi_{5}^{4}\left(A_{i} \varphi_{6} \varphi_{3}-B_{i} \varphi_{2}\right)\right)-\mu \varphi_{4}^{2} \varphi_{5}^{4}\left(B_{i} \varphi_{3}+A_{i} \varphi_{2} \varphi_{6}\right) \\
\frac{1}{2} \sqrt{\mu} \varphi_{4} \varphi_{5}^{3}\left(A_{i} \varphi_{6} \varphi_{3}-B_{i} \varphi_{2}\right)+\frac{3}{2} \mu\left(t_{1}-t_{0}\right) \varphi_{4}^{2} \varphi_{5}^{6}\left[t_{3} \varphi_{2} \varphi_{4}\left(A_{i} \varphi_{6} \varphi_{3}-B_{i} \varphi_{2}\right)+\right. \\
\left.\left.+B_{i} \varphi_{3}+A_{i} \varphi_{2} \varphi_{6}\right)\right] \\
\frac{\partial \varphi_{10+i}}{\partial t_{3}}=\sqrt{\mu} \varphi_{4}^{2} \varphi_{5}\left(\varphi_{3}-t_{3} \varphi_{2}^{2} \varphi_{4}\right)\left(A_{i} \varphi_{6} \varphi_{3}-B_{i} \varphi_{2}\right)- \\
-\sqrt{\mu} \varphi_{4} \varphi_{5}\left(\varphi_{2} \varphi_{3} \varphi_{4} B_{i}+\varphi_{2}^{2} \varphi_{4} \varphi_{6} A_{i}+t_{3} \varphi_{3} \varphi_{7} A_{i}\right) \\
\frac{\partial \varphi_{10+i}}{\partial t_{4}}=\sqrt{\mu} \varphi_{4}^{2} \varphi_{5}\left[t_{3} \varphi_{2} \varphi_{4}\left(A_{i} \varphi_{6} \varphi_{3}-B_{i} \varphi_{2}\right)-\left(B_{i} \varphi_{3}+A_{i} \varphi_{2} \varphi_{6}\right)\right]
\end{gathered}
$$

The second total polynomial system for the two body equations. We consider the total system for $\varphi_{13+i}=A_{i}, \varphi_{16+i}=B_{i}, i=1,2,3$, as functions of elements $t_{5}=i$, $t_{6}=\Omega, t_{7}=\omega$. If, in addition to these auxiliary functions (see (14)), four more functions

$$
\begin{gathered}
\varphi_{20}=A_{4}=\sin \omega \cos i, \quad \varphi_{21}=B_{4}=\cos \omega \cos i, \\
\varphi_{22}=A_{5}=\sin \Omega, \quad \varphi_{23}=B_{5}=\cos \Omega
\end{gathered}
$$

are introduced, then the desired total system will be written in the form

$$
\begin{aligned}
\frac{\partial \varphi_{14}}{\partial t_{6}} & =-\varphi_{15}, & \frac{\partial \varphi_{14}}{\partial t_{7}} & =-\varphi_{17}, & & \frac{\partial \varphi_{14}}{\partial t_{5}}=\varphi_{16} \varphi_{22}, \\
\frac{\partial \varphi_{17}}{\partial t_{6}} & =-\varphi_{18}, & \frac{\partial \varphi_{17}}{\partial t_{7}} & =\varphi_{14}, & & \frac{\partial \varphi_{17}}{\partial t_{5}}=\varphi_{19} \varphi_{22}, \\
\frac{\partial \varphi_{15}}{\partial t_{6}} & =\varphi_{14}, & \frac{\partial \varphi_{15}}{\partial t_{7}} & =-\varphi_{18}, & \frac{\partial \varphi_{15}}{\partial t_{5}} & =-\varphi_{16} \varphi_{23}, \\
\frac{\partial \varphi_{18}}{\partial t_{6}} & =\varphi_{17}, & \frac{\partial \varphi_{18}}{\partial t_{7}} & =\varphi_{15}, & & \frac{\partial \varphi_{18}}{\partial t_{5}}=-\varphi_{19} \varphi_{23} \\
\frac{\partial \varphi_{16}}{\partial t_{6}} & =0, & \frac{\partial \varphi_{16}}{\partial t_{7}} & =-\varphi_{19}, & \frac{\partial \varphi_{16}}{\partial t_{5}} & =\varphi_{21}, \\
\frac{\partial \varphi_{19}}{\partial t_{6}} & =0, & \frac{\partial \varphi_{19}}{\partial t_{7}} & =\varphi_{16}, & & \frac{\partial \varphi_{19}}{\partial t_{5}}=\varphi_{20}, \\
\frac{\partial \varphi_{20}}{\partial t_{6}} & =0, & \frac{\partial \varphi_{20}}{\partial t_{7}} & =\varphi_{21}, & \frac{\partial \varphi_{20}}{\partial t_{5}} & =-\varphi_{19}, \\
\frac{\partial \varphi_{21}}{\partial t_{6}} & =0, & \frac{\partial \varphi_{21}}{\partial t_{7}} & =-\varphi_{20}, & \frac{\partial \varphi_{21}}{\partial t_{5}} & =-\varphi_{16}, \\
\frac{\partial \varphi_{22}}{\partial t_{6}} & =\varphi_{23}, & \frac{\partial \varphi_{22}}{\partial t_{7}} & =0, & \frac{\partial \varphi_{22}}{\partial t_{5}} & =0 \\
\frac{\partial \varphi_{23}}{\partial t_{6}} & =-\varphi_{22}, & \frac{\partial \varphi_{23}}{\partial t_{7}} & =0, & \frac{\partial \varphi_{23}}{\partial t_{5}} & =0 .
\end{aligned}
$$


Conclusion. The main result of this article is the local guaranteed a priori error estimate (12) for the solution of the Cauchy problem (3) for the total linear system of partial differential equations using the Taylor series method (see consequently: equations (3), designations (4), formulas to the Taylor series method (5), inequality (8), scaling transformation (9) (with (10)), designations (11), inequality (12), the Perron's theorem, the Remarks 1,2, inequality (12), and item just after Remark 2). In the final part of the article, four examples of total systems of partial differential equations to the well-known two-body problem are proposed: two of them are related to the Kepler equation, one to the motion of a point in the orbit plane, and the last to the motion of the orbit plane.

\section{References}

1. Gaishun I. V. Vpolne razreshimiye mnogomerniye differentsialniye uravneniya [Completely solvable multidimensional differential equations]. Minsk, Belarus, Nauka i Technika Publ., 1983,272 p. (In Russian)

2. Babadzanjanz L. K. Metod dopolnitelnih peremennih [The additional variables method]. Vestnik of Saint Petersburg University. Series 10. Applied Mathematics. Computer Science. Control Processes, 2010, iss. 4, pp. 3-11. (In Russian)

3. Babadzanjanz L. K., Bregman K. M. Algorithm metoda dopolnitelnih peremennih [Algorithm of the additional variables method]. Vestnik of Saint Petersburg University. Series 10. Applied Mathematics. Computer Science. Control Processes, 2012, iss. 2, pp. 3-12. (In Russian)

4. Abad A., Barrio R., Blesa F., Rodriguez M. Breaking the limits: the Taylor series method. Appl. Math. and Computation, 2011, vol. 217, iss. 20, pp. 7940-7954.

5. Alesova I. M., Babadzanjanz L. K., Pototskaya I. Yu., Pupysheva Yu. Yu., Saakyan A. T. Highprecision numerical integration of equations in dynamics. International scientific conference on mechanics. The Eighth Polyakhov's Reading, Saint Petersburg, AIP Conference Proceedings. Saint Petersburg, 2018, vol. 1959, pp. 1-4, 080005. https://doi.org/10.1063/1.5034722

6. Babadzanjanz L. K. Metod ryadov Taylora [The Taylor series method]. Vestnik of Saint Petersburg University. Series 10. Applied Mathematics. Computer Science. Control Processes, 2010, iss. 3, pp. 13-29. (In Russian)

7. Berz M. Cosy infinity version 8 reference manual. Technical Report MSUCL-1088. Michigan, Michigan National Superconducting Cyclotron Lab., Michigan State University Press, 2003, 695 p.

8. Berz M., Bischof C., Corliss G. F., Griewank A. Computational differentiation: techniques, applications, and tools. Philadelphia, Society for Industrial and Applied Mathematics Publ., 1997,424 p.

9. Babadzanjanz L. K., Pototskaya I. Yu., Pupysheva Yu. Yu. Error estimates for numerical integration of ODEs in the minimax formulation. 2017 Constructive Nonsmooth Analysis and Related Topics (Dedicated to the memory of V. F. Demyanov), CNSA 2017. Proceedings. Saint Petersburg, Russia, 2017, pp. 1-4. https://doi.org/10.1109/CNSA.2017.7973932

10. Babadzanjanz L. K., Bol'shakov A. I. Primenenie metoda ryadov Taylora dlya resheniya obiknovennih differentsialnih uravneniy [Implementation of the Taylor series method for solving ordinary differential equations]. Vychisl. Metody Programm. [Computational programming methods], 2012, vol. 13, iss. 4, pp. 497-510. (In Russian)

11. Babadzanjanz L. K., Pupychev Yu. A., Pupycheva Yu. Yu. Classicheskaya mechanica [Classical Mechanics]. Saint Petersburg, Saint Petersburg State University Press, 2007, 240 p. Available at: http://www.apmath.spbu.ru/ru/staff/babadzhanyants/publ/publ36.pdf) (accessed: 14.07.2019). (In Russian)

12. Bellman R. Introduction to matrix analysis. 2nd ed. Philadelphia, Society for Industrial and Applied Mathematics Publ., 1997, 403 p.

13. Guntmacher F. R. The theory of matrices. In 2 vol. New York, Chelsea, 1960, 374 p.

14. Wilkinson J. N. The algebraic eigenvalue problem. Oxford, Clarendon Press, 1965, $564 \mathrm{p}$.

15. Ostrowski A. M. Solution of equations and systems of equations. 2nd ed. Pure and Applied Mathematics: A Series of Monographs and Textbooks. Elsevier, Acad. Press, vol. 9, 1966, 352 p.

16. Bregman K. M. Matematicheskie modeli vozmuschennogo dvighenia $v$ tsentral'nyh poliah [Mathematical models to perturbed motion in central fields]. PhD thesis, Saint Petersburg, Saint Petersburg State University Press, 2014, 145 p. (In Russian)

17. Babadzanjanz L. K., Bregman A. M., Bregman K. M., Kasikova P. V., Petrosyan L. A. Polniye systemy uravneniy $\mathrm{v}$ zadache dvuh tel [Total systems of equations to the two-body problem]. Engineering 
sciences - from theory to applications. Coll. papers on LXI Intern. conference, no. 8(56). Novosibirsk, SIBAK Publ., 2016, pp. 13-21. (In Russian)

Received: July 27, 2019.

Accepted: May 28, 2020.

Authors' information:

Levon K. Babadzanjanz - Dr. Sci. in Physics and Mathematics, Professor; levon-lkb@yandex.ru

Irina Yu. Pototskaya - PhD in Physics and Mathematics, Associate Professor; irinapototskaya@yandex.ru

Yulia Yu. Pupysheva - PhD in Physics and Mathematics, Associate Professor; j_poupycheva@mail.ru

\title{
Оценки в методе рядов Тейлора для линейных полных УрЧП
}

\author{
Л. К. Бабаджаняни, И. Ю. Поточкал, Ю. Ю. Пупъщева
}

Санкт-Петербургский государственный университет, Российская Федерация, 199034, Санкт-Петербург, Университетская наб., 7-9

Для цитирования: Babadzanjanz L. K., Pototskaya I. Yu., Pupysheva Yu. Yu. Estimates for Taylor series method to linear total systems of PDEs // Вестник Санкт-Петербургского университета. Прикладная математика. Информатика. Процессы управления. 2020. Т. 16. Вып. 2. С. 112-120. https://doi.org/10.21638/11701/spbu10.2020.203

Большое количество обыкновенных дифференциальных уравнений (ОДУ) можно свести к полиномиальной форме. Как было показано в ряде работ различных авторов, одним из лучших методов численного решения задачи начального приближения для таких систем ОДУ является метод рядов Тейлора. В данной работе рассматривается применение этого метода к решению задачи Коши для полной линейной системы дифференциальных уравнений в частных производных. Для обоснования эффективности подобного подхода формулируется и доказывается теорема о точности решения этой задачи методом рядов Тейлора. В последней части статьи приводятся четыре примера, иллюстрирующих алгоритм применения метода Тейлора в задачах небесной механики. Рассматриваются полные уравнения в частных производных, описывающие задачу двух тел. Первые две задачи относятся к уравнениям Кеплера. Третья задача описывает движение точки в плоскости орбиты. Последняя задача касается движения самой плоскости орбиты.

Ключевые слова: метод рядов Тейлора, полные линейные системы УрЧП, полиномиальные системы, численное интегрирование систем УрЧП.

Контактная информация:

Бабаджаняни, Левон Константинович - д-р физ.-мат. наук, проф.; levon-lkb@yandex.ru

Поточкая Ирина Юръевна - канд. физ.-мат. наук, доц.; irinapototskaya@yandex.ru

Пупышева Юлия Юръевна - канд. физ.-мат. наук, доц.; j_poupycheva@mail.ru 